\title{
Percepção de profissionais de saúde em relação ao cuidado a pessoas idosas institucionalizadas
}

\author{
Thepercedion of hedth professiondstonardthecreof instittiondized ddaly
}

Diéssica Roggia Piexak

Paula Hübner Freitas ${ }^{2}$

Dirce Stein Backes ${ }^{3}$

Claudete Moreschi ${ }^{4}$

Carla Lizandra de Lima Ferreira ${ }^{3}$

Martha Helena Teixeira de Souza ${ }^{3}$

\section{Resumo}

Objetivo: conhecer o significado que profissionais de saúde atribuem ao cuidado de pessoas idosas institucionalizadas. Método: pesquisa qualitativa, exploratóriodescritiva, realizada em uma instituição de longa permanência para idosos localizada na região central do Rio Grande do Sul. Participaram do estudo 14 profissionais de saúde: enfermeiros, farmacêutico, fisioterapeuta, médico, nutricionista e técnicos de enfermagem. Os dados foram coletados entre agosto e setembro de 2009, por meio de um questionário semiestruturado. Resultados: os dados analisados resultaram em três categorias: Cuidando de pessoas idosas, Percebendo a equipe multidisciplinar e Enfrentando a nova realidade. Conclusão: o cuidado de pessoas idosas institucionalizadas constitui um desafio crescente para os profissionais de saúde, a ser superado por meio do trabalho multidisciplinar.

\section{Abstract}

Objective: To know the meaning attributed by health professionals to the care of institutionalized elderly. Method: Qualitative, exploratory-descriptive research, carried out in a long-term care institution for the elderly located in central Rio Grande do Sul state. Fourteen health professionals took part in the study, including nurses, pharmacist, physiotherapist, doctor, nutritionist, and nursing technicians. Data were collected between August and September 2009, through a semi-structured questionnaire. Results: Data analysis has resulted in three categories: Caring for the elderly, Realizing the multidisciplinary team, and Facing the new reality. Conclusion: the care of the institutionalized elderly is a growing challenge for health professionals, and it needs to be coped with through multidisciplinary work.

\footnotetext{
Programa de Pós-Graduação em Enfermagem, Universidade Federal do Rio Grande. Rio Grande, RS, Brasil.

2 Programa de Pós-Graduação em Enfermagem, Universidade Federal de Santa Maria. Santa Maria, RS, Brasil.

3 Curso de Enfermagem, Centro Universitário Franciscano. Santa Maria, RS, Brasil.

4 Programa de Pós-Graduação em Ambiente e Desenvolvimento, Cento Universitário UNIVATES. Lageado, RS, Brasil.
}

\section{Palavras-chave:}

Enfermagem Geriátrica. Equipe de Assistência ao Paciente. Instituição de Longa Permanência para Idosos.
Key words: Geriatric Nursing. Patient Care Team. Homes for the Aged.Longterm care institution for the elderly. 


\section{INTRODUÇÃO}

Envelhecer é um processo natural que caracteriza uma etapa da vida do ser humano, quando ocorrem mudanças físicas, psicológicas e sociais que acometem de forma particular cada ser humano. ${ }^{1}$ Segundo afirmações em documentos da Organização Mundial da Saúde (OMS), ${ }^{2}$ da Política Nacional do Idoso (Lei no 8.842/1994) ${ }^{3}$ e do próprio Estatuto do Idoso (Lei $\left.\mathrm{n}^{\mathrm{o}} 10.741 / 2003\right),{ }^{4}$ considera-se idosa a pessoa com 60 anos ou mais nos países em desenvolvimento, como é o caso do Brasil. O envelhecimento, como processo irreversível, necessita ser mais bem compreendido, especialmente no Brasil, que vem apresentando número crescente de pessoas idosas e modificando gradativamente sua pirâmide populacional. ${ }^{5}$

O envelhecimento populacional é um fenômeno universal, característico dos países desenvolvidos como também, de modo crescente, daqueles em desenvolvimento. A Europa é a região onde a população é mais velha - as pessoas idosas representam $21 \%$ do total, enquanto as crianças são 15\%. As expectativas na Europa são que essas pessoas constituirão $35 \%$ do total da população em 2050 e que na América do Norte, atualmente a segunda região mais velha do planeta, com $17 \%$, alcance $27 \%$ em 2050. ${ }^{6}$

É notório que o envelhecimento de uma população traz desafios, visto que implica repensar das políticas sociais e de saúde. $\mathrm{Na}$ Europa foi necessário planejar novas estratégias frente ao crescente número de pessoas idosas, tais como: a Estratégia Europeia do Emprego, Políticas de Proteção Social, Políticas de Saúde, prestação de cuidados a pessoas idosas e investigação nestas áreas e políticas de luta contra a discriminação e a exclusão social, para poder garantir qualidade de vida a essas pessoas. ${ }^{6}$

No Brasil, esta realidade não será muito diferente. Conforme dados do Instituto Brasileiro de Geografia e Estatística (IBGE), no ano de 2020, a população de idosos poderá alcançar e até mesmo ultrapassar 30 milhões de pessoas (aproximadamente 13\% da população brasileira). ${ }^{7}$
No recente censo de 2010, o número de pessoas com 60 anos ou mais é de 20.622.018, enquanto que em 2000, o número era de 14.536.029, contra 10.722 .705 em $1991 .^{8}$

Nessa perspectiva, observa-se o crescente número de pessoas idosas, que necessitam de cuidados diferenciados e prestados por profissionais qualificados. Torna-se indispensável que profissionais de saúde estejam capacitados para cuidar dessa população. Esses profissionais precisarão conhecer o contexto em que se encontram essas pessoas. Algumas pessoas idosas poderão encontrar-se em instituições de longa permanência para idosos (ILPI), as quais podem ser públicas ou particulares e com ou sem ajuda financeira do governo.

As ILPIs devem zelar e acolher as pessoas idosas de forma humanizada, fornecendolhes alimentação, moradia, cuidados com a higiene pessoal e saúde adequadas. Devem também proporcionar atividades recreativas, lúdicas, esportivas, manuais e sociais, as quais possibilitam um envelhecimento com cidadania, dignidade e liberdade. ${ }^{?}$

A Lei n ${ }^{\circ}$ 8.842/94 criou o Conselho Nacional do Idoso, responsável pela viabilização do convívio, integração e ocupação da pessoa idosa na sociedade, por meio de sua participação na formulação das políticas públicas, projetos e planos destinados à sua faixa etária. Na Política Nacional do Idoso (PNI), sancionada pela Lei $\mathrm{n}^{\mathrm{o}} 8.842 / 94$ e regulamentada pelo Decreto $\mathrm{n}^{\circ}$ 1.948/96, destaca que a família, a sociedade e o Estado têm o dever de assegurar a pessoa idosa todos os direitos de cidadania, garantindo sua participação na comunidade, defendendo sua dignidade, bem-estar e o direito à vida. Além disso, a pessoa idosa deve ser o principal agente e o destinatário das transformações a serem efetivas através dessa política. ${ }^{3}$ Seguindo as diretrizes da PNI, foi revista a proposta direcionada à saúde da pessoa idosa, com a aprovação da Política Nacional de Saúde da Pessoa Idosa (PNSPI), regulamentada pela Portaria GM n ${ }^{\circ} 2.528$, de 19 de outubro do referido ano, no contexto do Pacto em Defesa do Sistema Único de Saúde (SUS), do pacto em Defesa da Vida e do Pacto de Gestão.' 
$\mathrm{O}$ aumento da expectativa de vida, especificamente no Rio Grande do Sul, desencadeou a necessidade de revisão do modelo assistencial, adequando a oferta de serviços ao perfil da população, que passa a necessitar de acompanhamento continuado pelas equipes de saúde. Para viabilizar esta mudança no sistema de saúde, foi aprovada em 2005 a Política Estadual de Saúde do Idoso (Resolução CIB/RS no 227/05). ${ }^{10}$

Também foi criado o Programa RS Amigo do Idoso, que tem por objetivo desenvolver ações que propiciem a inclusão social das pessoas idosas, respeitando a heterogeneidade $\mathrm{e}$ as diversidades cultural, regional, étnico-racial, de gênero e de orientação sexual, como forma de garantir o exercício da cidadania, estimulando a emancipação, autonomia e independência nas atividades cotidianas, bem como as potencialidades e habilidades. ${ }^{10}$

O envelhecimento pode exigir cuidados para a manutenção da vida cotidiana da pessoa idosa, geralmente desempenhado por familiares que costumam assumir o papel de cuidadores, por terem uma responsabilidade culturalmente definida e/ou por vínculo afetivo. ${ }^{11} \mathrm{O}$ cuidado de pessoas idosas também pode ser desempenhado por pessoas contratadas pela família e/ou de sua rede social - o que nem sempre implica preparo técnico para o exercício da função de cuidador, pois esta demanda capacitação e nem sempre os trabalhadores recebem orientação e atenção. Por isso, salienta-se a importância merecida, pela carga emocional envolvida no cuidado. ${ }^{12}$

Torna-se cada vez mais importante a reflexão acerca da criação e/ou aperfeiçoamento de ILPIs destinadas a prestar cuidados à pessoa idosa, a qual depende de cuidados diferenciados, em função das condições sociais, mentais, físicas e afetivas nas quais vivem. Além da qualificação dos profissionais de saúde para enfrentar essa realidade, é preciso ampliar as políticas sociais para melhor atender as necessidades das pessoas idosas.

Um dos maiores desafios do cuidado multidisciplinar à pessoa idosa é propiciar que múltiplas áreas do saber ajam conjuntamente para um bem comum, ou seja, atender a pessoa idosa nas suas particularidades, tendo um olhar multidimensional e buscando prevenir agravos. ${ }^{13}$ Como algumas vezes as pessoas idosas precisam de ajuda, o mais significativo para elas é alguém que lhes ofereça essa ajuda como fonte de seu cuidado. Isso corrobora a precisão de estruturar uma equipe multidisciplinar qualificada, com amplo conhecimento geriátrico e gerontológico, dando ênfase aos aspectos biopsico-socioculturais. ${ }^{14}$

Este estudo apresenta como questão norteadora: qual o significado que profissionais de saúde atribuem ao cuidado de pessoas idosas institucionalizadas? Tem como objetivo conhecer o significado que profissionais de saúde atribuem ao cuidado de pessoas idosas institucionalizadas.

\section{METODOLOGIA}

Pesquisa qualitativa, exploratório-descritiva, que buscou conhecer o significado que profissionais de saúde atribuem ao cuidado de pessoas idosas institucionalizadas. Foi realizada em uma instituição de longa permanência para idosos (ILPI), localizada na região central do estado do Rio Grande do Sul. Os sujeitos do estudo foram 14 profissionais da equipe multidisciplinar de saúde, dentre os quais enfermeiros, farmacêutico, fisioterapeuta, médico, nutricionista e técnicos de enfermagem. Foram convidados todos os profissionais que atuam na ILPI, das diferentes categorias profissionais da área da saúde, e 14 tiveram disponibilidade e aceitaram o convite.

Para a coleta de dados, realizada entre agosto e setembro de 2009, foi aplicado um questionário semiestruturado, composto por sete questões abertas e fechadas. Foram garantidos o sigilo e o anonimato de cada participante da pesquisa após a anuência e assinatura do termo de consentimento livre e esclarecido. Cada participante foi identificado com a letra "P" (profissional), seguida de um número correspondente à ordem dos questionários respondidos. A coleta de dados cessou quando, ao codificar e analisar os dados, verificou-se que não houve surgimento de novas propriedades. 
Os dados foram analisados por categorização, com base no método de análise de conteúdo, ${ }^{15}$ constituído de três momentos. O primeiro consiste em uma frequência com identificação dos principais significados que os profissionais de saúde atribuem ao cuidado de pessoas idosas institucionalizadas. O segundo analisa o conteúdo que identifica as categorias que emergiram a partir dos dados coletados. O terceiro refere-se à interpretação das categorias, compreendendo o significado que os profissionais de saúde atribuem ao cuidado de pessoas idosas institucionalizadas.

Foi delimitada a unidade complexa, traduzida na expressão "Interligando saberes entre os diversos profissionais de saúde para o cuidado integral à pessoa idosa" e nos apoiamos nos escritos de Edgar Morin, autor da Complexidade, para discussão dos resultados ${ }^{16-21}$.

Para atender aos critérios éticos, foram seguidas as recomendações da Resolução $\mathrm{n}^{\circ}$ 196/96, do Conselho Nacional de Saúde. ${ }^{22}$ O protocolo de pesquisa foi aprovado pelo Parecer $n^{\circ}$ 092/2009/2 do Comitê de Ética em Pesquisa com Seres Humanos do Centro Universitário Franciscano (UNIFRA).

\section{RESULTADOS E DISCUSSÃO}

A partir dos resultados codificados e analisados, emergiu a unidade complexa "Interligando saberes entre os diversos profissionais de saúde para o cuidado integral a pessoa idosa", que foi decodificada nas seguintes categorias: Cuidando de pessoas idosas, Percebendo a equipe multidisciplinar e Enfrentando a nova realidade.

Interligando saberes entre os diversos profissionais de saúde para o cuidado integral à pessoa idosa

Ao analisar as respostas dos participantes, compreendemos como imprescíndivel o trabalho multidisciplinar, o qual poderá possibilitar um cuidado integral à pessoa idosa, valorizando o ser humano multidimensional que necessita de diferentes cuidados.
Para Edgar Morin, é preciso compreender o ser humano como um todo integrado. $\mathrm{O}$ autor defende o pensamento complexo como aquele que não separa, mas une e busca as relações necessárias e interdependentes de todos os aspectos da vida humana. É um pensamento integrador dos diferentes modos de pensar, opondo-se aos mecanismos reducionistas, disjuntivos e simplificadores. Por meio desse pensamento, são consideradas todas as influências recebidas, externas e internas, e ainda a incerteza e a contradição, sem deixar de conviver com a solidariedade dos fenômenos existentes. ${ }^{16,17}$

A complexidade é capaz de reunir, contextualizar, globalizar, mas ao mesmo tempo pode reconhecer o singular, o individual, o concreto. ${ }^{16}$ Portanto, cuidar a partir do olhar da complexidade implica compreender o ser humano na sua multidimensionalidade, integrando-o no seu contexto familiar, cultural, histórico e social.

O processo de envelhecimento humano é, na maioria das vezes, rejeitado pelo ser humano, porque o mesmo acaba rejeitando a morte, logo tende a rejeitar também a velhice, talvez por ser a fase da vida que mais se aproxime da morte. ${ }^{18}$ São os profissionais da equipe mutidisciplinar que irão desenvolver, juntos, estratégias para ajudar as pessoas idosas a enfrentar o desafio de aceitação do envelhecimento, o seu curso de vida, a sua totalidade e sua integração consigo próprio, com o outro e com o cosmo.

Para Edgar Morin, a origem, o motor do processo de envelhecer é difícil de se perceber, visto que o caráter patológico da velhice se manisfesta em três planos: no social; na percepção de que a velhice sadia é patológica enquanto velhice em si; e na própria morte, que é patológica e é aproximada pela velhice. Ele aceita que a velhice e a morte estão inscritas na herança genética humana e que são "coisas normais e naturais, porque uma e outra são universais e não sofrem qualquer exceção entre os 'mortais"'(p.320). ${ }^{19}$

Torna-se possível e necessária a interligação dos saberes entre os diversos profissionais de saúde para propiciar um envelhecimento saúdavel, 
ao refletir sobre a multidimensionalidade que cerca o processo de envelhecer.

\section{Cuidando de pessoas idosas}

Os profissionais da instituição de longa permanência para idosos investigada atuam há mais de dois anos com pessoas idosas. Eles procuram participar de estudos direcionados a capacitações em Geriatria e Gerontologia.

\section{[...] através de estudo pessoal e curso de especialização} (P1).

[...] a parir de leituras de pesquisas relacionadas ao tema idosos (P13).

Cursos da faculdade, palestras, seminários, jornadas acadêmicas (P14).

Considerando ser esta pesquisa realizada por enfermeiros, discutimos sobre o objeto de trabalho dessa displina, que é o cuidado humano, o qual é o fundamento da ciência Enfermagem, e acreditamos que cuidar significa "empreender comportamentos e ações que envolvam conhecimentos, valores, habilidades e atitudes, no sentido de favorecer as potencialidades das pessoas para manter ou melhorar a condição humana no processo de viver e morrer" (p.127). ${ }^{23}$ A equipe de enfermagem presta o seu cuidado diariamente às pessoas idosas da ILPI, mas além do cuidado da enfermagem, esses idosos precisam de um cuidado multidisciplinar. Por meio de uma equipe multidisciplinar capacitada, as pessoas idosas poderão ter suas diferentes necessidades de saúde atendidas. As ILPIs devem cumprir essa dupla função de ser um lugar para as pessoas idosas viverem e proporcionar os cuidados de que necessitam. ${ }^{24}$

As oportunidades de capacitações visam a contribuir para que esses profissionais de saúde tenham melhor preparo para enfrentar os atuais desafios e os que poderão surgir em decorrência do crescente número de pessoas com mais de 60 anos. É relevante que tais profissionais estejam aptos para efetuar ações/estratégias adequadas e implementar um cuidado integral a pessoa idosa e seus familiares, atuando na prevenção de doenças e promoção, recuperação e manutenção da vida.

$\mathrm{Na}$ pergunta direcionada aos profissionais investigados - "Desde que vocêiniciou a trabalhar com idosos já adquiriu algum conhecimento diferenciado para cuidar dos mesmos?" -, verificou-se que os que responderam "sim" indicaram acreditar que o cuidado depende quase que exclusivamente da experiência, desconsiderando a necessidade de qualificação para cuidar da pessoa idosa.

[...] conhecendo o perfil de cada idoso cuidado, e assim aperfeiçoando os cuidados (P10).

[...] através do cuidado direto com os mesmos, ao longo deste tempo (P2).

Através da prática profissional (P11).

A qualificação profissional e a ampliação de pesquisas científicas na área gerontológica são imprescindíveis para se cuidar da pessoa idosa. Os profissionais de saúde que trabalham com idosos deverão perceber a necessidade de qualificação, para que oportunizem um cuidado específico e adequado.

Segundo a PNSPI: há uma escassez de equipes multiprofissionais e interdisciplinares com conhecimento em envelhecimento e saúde da pessoa idosa. O crescimento demográfico da população idosa brasileira exige a preparação adequada para atender a suas demandas. Essa preparação envolve diferentes aspectos que dizem respeito a adequação ambiental e provimento de recursos materiais e humanos capacitados, definição e implementação de ações de saúde específicas. A prática de cuidados às pessoas idosas exige abordagem global, multidimensional e interdisciplinar, que leve em conta a interação entre os fatores físicos, psicológicos e sociais, os quais influenciam na saúde dos idosos. ${ }^{?}$

Os indivíduos fazem a sociedade, que faz os indivíduos. Somos produtos e produtores, ao mesmo tempo. Assim, também quando se considera o fenômeno social, são as interações 
entre indivíduos que produzem a sociedade, mas a sociedade, com sua cultura e normas, retroage sobre os indivíduos e os produz enquanto indivíduos sociais dotados de uma cultura. Indivíduos e sociedade se coproduzem num circuito recursivo permanente. ${ }^{20}$ As instituições de ensino, os profissionais de saúde e as pessoas idosas precisam se comunicar e se interligar, para que juntos possam traçar estratégias de enfrentamento dos desafios que possam surgir com o envelhecimento.

Ocuidado não éalgo independente de nós, pelo contrário, é vivido e se estrutura em nós mesmos. "O cuidado possui uma dimensão ontológica que entra na constituição do ser humano. É um modo-de-ser singular do homem e da mulher. Sem cuidado, deixamos de ser humanos" (p.89). ${ }^{25}$ Nas respostas, ao questionarmos "Para você o que é cuidar de idosos?", evidencia-se que os profissionais de saúde atribuem fatores subjetivos como relevantes ao cuidar:

É muito gratificante pelo carinho que vem em retorno, é ter paciência, é lutar para que tenham um envelhecimento saudável [...] (P1).

É dar amor [...] (P11).

\section{Percebendo a equipe multidisciplinar}

Nas respostas dos profissionais de saúde, podese perceber que eles acreditam que é essencial a formação de uma equipe multidisciplinar para cuidar de idosos. Os idosos apresentam diversas necessidades de cuidados e que quanto maior o número de profissionais de saúde capacitados e trabalhando em equipe, melhor poderá ser o cuidado prestado. Importante também considerar que a multidisciplinaridade pode estimular o surgimento da interdisciplinaridade, que é em si a interligação dos saberes.

O idoso apresenta um conjunto de necessidades (físicas, psicológicas, sociais) que demandam muito esforço, conbecimento e diferentes profissionais trabalhando juntos (P1).
O princípio "Hologrâmico", inspirado no holograma, em que cada ponto contém a quase totalidade da informação do objeto que ele representa, evidencia que não apenas a parte está inscrita no todo, como o todo está inscrito na parte. A sociedade está presente em cada indivíduo, como todo, através de sua linguagem, sua cultura, suas normas. ${ }^{20}$ Assim, a união dos saberes, que atravessa a multi e a interdisciplinaridade, deve tornar evidentes o contexto, o global, o multidimensional e o complexo, princípios para um conhecimento pertinente. ${ }^{21}$

Os profissionais de saúde que atuam na ILPI direcionaram seus depoimentos à necessidade da multidisciplinaridade e apresentando um pensamento integrador visualizando a necessidade da interligação dos saberes, ocorrida por meio da inter e transdisciplinaridade. A interdisciplinaridade é percebida quando existe a possibilidade de transformação da realidade em que se atua, procurando-se colocar as partes em relação ao seu significado no todo. Pressupõe ausência de preconceito teórico e que termina sendo um modo de se compreender o mundo, é movimento, algo que se vive. ${ }^{26}$

\section{Enfrentando a nova realidade}

Na ILPI, percebe-se um dos desafios que os profissionais de saúde enfrentam: o abandono das pessoas idosas. As famílias, por inúmeros motivos, acabam deixando seus idosos na ILPI e muitos nunca mais voltam para vê-los. Essa realidade é preocupante para os profissionais de saúde, como relatada nos depoimentos seguintes:

[...] muitos destes idosos já trabalharam muito para cuidarem de suas famílias e no presente estão abandonados por seus familiares (P2).

[...] às vezes eles [os idosos] não entendem o abandono da familia, assim temos que prestar assistência a esses idosos, fazendo com que se sintam mais felizes (P10).

A família é entendida como rede social de apoio, constituída por um grupo hierarquizado de pessoas que mantêm entre si laços e relações 
de dar e receber, onde se almeja uma atmosfera afetiva comum, de aquisição de competência e de interação entre seus membros. ${ }^{27,28} \mathrm{O}$ cuidado familiar à pessoa idosa é um aspecto importante. Segundo o Estatuto do Idoso, no Art. 3. Parágrafo Único, Ponto V: "priorização do atendimento ao idoso por sua própria família, em detrimento do atendimento asilar, exceto dos que não a possuam ou careçam de condições de manutenção da própria sobrevivência". ${ }^{4}$ Por conta do reduzido número de membros familiares e dificuldades surgidas no mundo moderno, algumas famílias necessitam das ILPIs para acolher seus idosos.

A pessoa idosa institucionalizada deve ter o contato familiar, pois o mesmo permite que se mantenham próximos ao ambiente familiar, preservando assim seus valores e mantendo sua autoestima. ${ }^{29}$ Uma constante preocupação dos profissionais de saúde da ILPI é procurar motivar os familiares a serem mais ativos no cuidado da pessoa idosa, estabelecendo vínculo entre pessoa idosa, família e profissional de saúde.

\section{CONSIDERAÇÕES FINAIS}

Com o objetivo de conhecer o significado que profissionais de saúde atribuem ao cuidado de pessoas idosas institucionalizadas, o presente estudo evidenciou a importância do trabalho multidisciplinar para um cuidado mais específico à pessoa idosa que se encontra em ILPI.

Apesar da importância da atuação da equipe multidisciplinar, membros desta equipe acreditam que o cuidado por longo tempo a esses idosos já é suficiente para o bom atendimento, não sendo de grande importância a realização de capacitações na área.

Os profissionais de saúde, entre eles o enfermeiro, precisam ampliar seu olhar na busca de novos caminhos que acompanhem o crescimento da população idosa em termos de atualização sobre o tema, visando cada vez mais a um atendimento condizente com esta realidade. Para tanto, é fundamental que procurem compreender a realidade complexa da pessoa idosa, inserindo neste contexto as questões sociais que incluem as causas do abandono que emergem nestas situações.

O estudo aponta o abandono das pessoas idosas como um problema real. Este fato sugere que novas pesquisas sejam desenvolvidas, buscando as causas desse abandono e uma possível atuação junto a essas famílias, fazendo com que diminuam os índices desta realidade.

Para amenizar os problemas e dificuldades encontrados no atendimento às pessoas idosas em uma ILPI e para prestar um cuidado eficaz a esta população, é preciso que os profissionais de saúde conheçam e adotem os objetivos da Política Nacional de Saúde da Pessoa Idosa, garantindo sua dignidade, seu bem-estar e o seu direito à vida.

Cabe ao enfermeiro e aos demais profissionais de saúde se engajarem no trabalho multidisciplinar, em busca de uma qualidade de vida para as pessoas idosas.

\section{REFERÊNCIAS}

1. Mendes MB, et al. A situação social do idoso no Brasil: uma breve consideração. Acta paulista de enfermagem. 2005; 18(4): 422-6.

2. Organização das Nações Unidas - ONU. Assembléia Mundial sobre envelhecimento: Resolução 39/125. Viena, 1982.

3. Brasil. Presidência da República. Casa Civil. Subchefia para Assuntos Jurídicos. Lei no 8.842 , de 4 de janeiro de 1994. Dispõe sobre a política nacional do idoso, cria o Conselho Nacional do Idoso e dá outras providências. Brasília (DF): 1994.
4. Brasil. Presidência da República. Casa Civil. Subchefia para Assuntos Jurídicos. Lei n 10.741, de $1^{\circ}$ de outubro de 2003. Dispõe sobre o Estatuto do Idoso e dá outras providências. Brasília (DF): 2003.

5. Santos SSC. O ensino da enfermagem gerontogeriátrica e a complexidade. Rev Esc Enferm USP. 2006; 40(2):228-35.

6. Comissão das Comunidades Européias. Uma Europa para todas as idades: Promover a prosperidade e a solidariedade entre as gerações. Bruxelas, COM (1999) 221 final, 1999. [acesso 2011 fev 05]. 
Disponível em: http://ec.europa.eu/employment_ social/social_situation/docs/com221_pt.pdf.

7. IBGE/Diretoria de Pesquisas. Censo Demográfico, 2000. Rio de Janeiro: IBGE; 2002.

8. IBGE [acesso 2010 jan21]. Disponível em: <http:// www.ibge.gov.br/brasil_em_sintese/default.htm>

9. Brasil. Ministério da Saúde. Secretaria de Atenção à Saúde. Departamento de Atenção Básica.

Envelhecimento e saúde da pessoa idosa. Brasília:

Ministério da Saúde; 2007. (Cadernos de Atenção Básica, n. 19).

10. Rio Grande do Sul. Secretaria da Saúde. Resolução CIB/RS 227/05: aprova a Política Estadual do Idoso. [acesso 2011 fev15]. Disponível em: http://www.saude.rs.gov.br/wsa/portal/index. jsp? $\mathrm{menu}=$ organograma\&cod $=42498$.

11. Cattani RB, Girardon-Perlini NMO. Cuidar do idoso doente no domicílio na voz de cuidadores familiares. Revista eletronica de enfermagem. 2004 maio-ago; 6 (2): 254-71.

12. Machado ALG, Freitas CHA, Jorge MSB. O fazer do cuidador familiar: significados e crenças. Rev Bras Enferm. 2007 set-out; 60(5): 530-4.

13. Carvalho Filho ET. Papaléo Netto MP. Geriatria: Fundamentos, Clínica e Terapêutica. São Paulo: Atheneu; 1994.

14. Nouwen HJM, Gaffney WJ. Envelhecer: a plenitude da vida. São Paulo: Paulinas; 2000.

15. Bardin L. Análise de conteúdo. 4 ed. Lisboa: Edições, 2009.

16. Morin E. A epistemologia da complexidade. In: Morin E, Le Moigne JL. A inteligência da complexidade. São Paulo (SP): Fundação Peirópolis: 2000.

17. ___._. Ciência com consciência. 14 ed. Rio de Janeiro: Bertrand Brasil; 2010.
18. _____. Por uma reforma do pensamento. In: PenaVega A, Nascimento EP. O pensar complexo: Edgar Morin e a crise da modernidade. Rio de Janeiro: Gramond; 1999.

19. ____. O homem e a morte. Rio de Janeiro: Imago, 1997.

20. ____. A Cabeça bem-feita: repensar a reforma, reformar o pensamento. 17. ed. Rio de Janeiro: Bertrand Brasil; 2010.

21. ______. Os sete saberes necessários à educação do futuro. São Paulo: Cortez; 2002.

22. Brasil. Conselho Nacional de Saúde. Resolução n. 196, de 10 de outubro de 1996. Diretrizes e normas regulamentadoras de pesquisa em seres humanos. Brasília: Ministério da Saúde; 1996.

23. Waldow VR. Cuidado humano: o resgate necessário. Porto Alegre (RS): Sagra Luzzatto; 1998.

24. Kane RA, Kane RL. Long-Term Care: principles, programs and policies. New York: Springer; 1987.

25. Boff L. Saber cuidar: ética do humano-compaixão pela terra. 10. ed. Petrópolis: Vozes; 2004.

26. Petraglia IC. Interdisciplinaridade: o cultivo do professor. São Paulo: Pioneira; 1993.

27. Pelzer MT, Fernandes MR. Apoiando a família que cuida de seu familiar idoso com demência. Texto \& contexto enfermagem / UFSC. 1997; 6: 339-4.

28. Montezuma CA, Freitas MC, Monteiro ARM. A família e o cuidado ao idoso dependente: estudo de caso. Revista eletronica de enfermagem. 2008;10(2):395-404. [acesso 2011 fev18]. Disponível em: <http://www.fen.ufg.br/revista/v10/n2/ v10n2a11.htm>.

29. Papaléo Netto M. Gerontologia: a velhice e o envelhecimento em visão globalizada. São Paulo: Atheneu; 2000. 